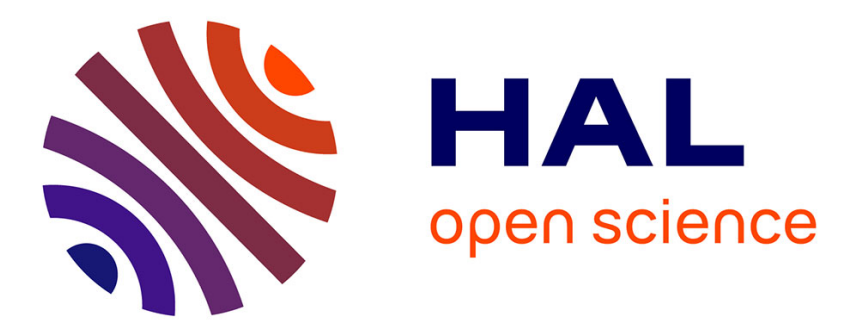

\title{
Shape Coexistence in Light Krypton Isotopes
}

\author{
A. Görgen, E. Clément, E. Bouchez, A. Chatillon, W. Korten, Y. Le Coz, Ch. \\ Theisen, C. Andreoiu, F. Becker, Bertram Blank, et al.
}

\section{To cite this version:}

A. Görgen, E. Clément, E. Bouchez, A. Chatillon, W. Korten, et al.. Shape Coexistence in Light Krypton Isotopes. Acta Physica Polonica B, 2005, 36, pp.1281. in2p3-00024007

\section{HAL Id: in2p3-00024007 https://hal.in2p3.fr/in2p3-00024007}

Submitted on 8 Jan 2007

HAL is a multi-disciplinary open access archive for the deposit and dissemination of scientific research documents, whether they are published or not. The documents may come from teaching and research institutions in France or abroad, or from public or private research centers.
L'archive ouverte pluridisciplinaire HAL, est destinée au dépôt et à la diffusion de documents scientifiques de niveau recherche, publiés ou non, émanant des établissements d'enseignement et de recherche français ou étrangers, des laboratoires publics ou privés. 


\title{
Shape coexistence in light Krypton isotopes
}

\author{
A. Görgen, ${ }^{1}$ E. Clément,${ }^{1}$ E. Bouchez, ${ }^{1}$ A. Chatillon,${ }^{1}$ \\ W. Korten, ${ }^{1}$ Y. Le Coz, ${ }^{1}$ Ch. Theisen, ${ }^{1}$ C. Andreoiu, ${ }^{2,3}$ \\ F. Becker,${ }^{4}$ B. Blank,${ }^{5}$ A. Bürger,${ }^{6}$ P. Butler,${ }^{2,7}$ \\ J.M. Casandjian,${ }^{8} \mathrm{~W}$. Catford,${ }^{9}$ T. Czosnyka,${ }^{10}$ P. Davies,${ }^{11}$ \\ S.P. Fox,${ }^{11}$ G. de France, ${ }^{8}$ G. Georgiev, ${ }^{7,8}$ J. Gerl, ${ }^{4}$ H. Hübel,${ }^{6}$ \\ J. Iwanicki ${ }^{10}$ D.G. Jenkins,${ }^{11}$ F. Johnston-Theasby,${ }^{11}$ P. Joshi,${ }^{11}$ \\ I. Matea, ${ }^{5,8}$ P. Napiorkowski, ${ }^{10}$ F. De Oliveira Santos, ${ }^{8}$ \\ G. Sletten,${ }^{12}$ C. Timis,${ }^{9}$ R. Wadsworth, ${ }^{11}$ M. Zielinska ${ }^{10}$ \\ ${ }^{1}$ DAPNIA/SPhN, CEA Saclay, F-91191 Gif-sur-Yvette, France \\ ${ }^{2}$ Oliver Lodge Laboratory, University of Liverpool, Liverpool, L69 7ZE, UK \\ ${ }^{3}$ Dept. of Physics, University of Guelph, Guelph, Ontario, N1G 2W1, Canada \\ ${ }^{4}$ Gesellschaft für Schwerionenforschung, D-64291 Darmstadt, Germany \\ ${ }^{5}$ Centre d'Etudes Nucléaires de Bordeaux-Gradignan, F-33175 Gradignan, France \\ ${ }^{6}$ HISKP, Universität Bonn, Nussallee 14-16, D-53115 Bonn, Germany \\ ${ }^{7}$ CERN, CH-1211 Geneva 23, Switzerland \\ ${ }^{8}$ GANIL, F-14076 Caen Cedex, France \\ ${ }^{9}$ Department of Physics, University of Surrey, Guildford, GU2 7XH, UK \\ ${ }^{10}$ Heavy Ion Laboratory, Warsaw University, Warsaw, PL-02097, Poland \\ ${ }^{11}$ Department of Physics, University of York, Heslington, York YO10 5DD, UK \\ ${ }^{12}$ Niels Bohr Institute, Blegdamsvej 17, DK-2100 Copenhagen Ø, Denmark
}

The light Krypton isotopes were studied in a series of Coulomb excitation experiments using radioactive beams at GANIL. The static quadrupole moments found in these experiments give firm experimental evidence for the shape coexistence scenario that is based on theoretical calculations and on the systematics of low-lying excited $0^{+}$states.

PACS numbers: 21.10.Ky; 23.20.Js; 25.70.De; 27.50.+e

\section{Introduction}

The deformation of nuclei between closed shells is governed by a delicate interplay of macroscopic and microscopic effects. Prolate deformations occur far more abundantly than oblate shapes, and there are only few examples throughout the nuclear chart that are predicted to have a large oblate ground-state deformation. These are in particular the neutron-rich isotopes 

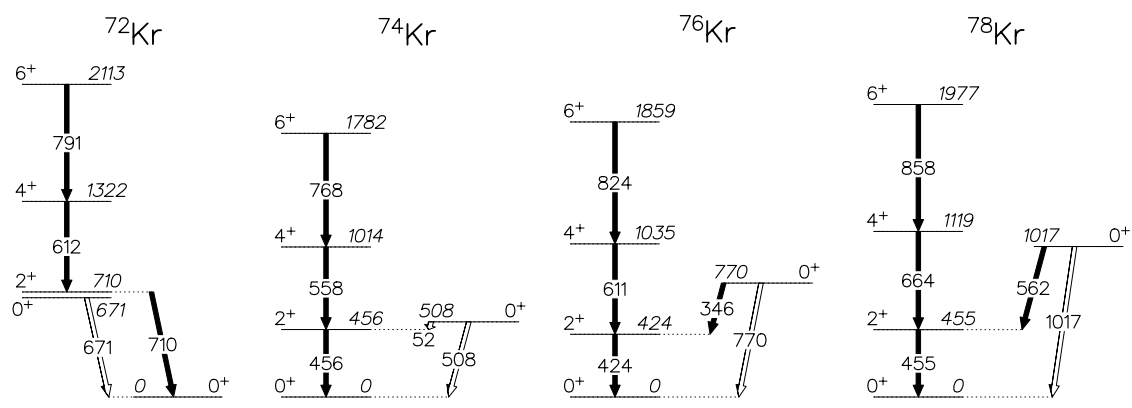

Fig. 1. Partial level schemes of the light even-even Krypton isotopes illustrating the systematics of low-lying $0^{+}$states. States with proposed prolate configuration are shown on the left, those with proposed oblate configuration on the right of each level scheme. Transitions proceeding via conversion electrons are shown as open arrows.

around Mo and the proton-rich Se and $\mathrm{Kr}$ isotopes near the $N=Z$ line. States of prolate and oblate shape are predicted to coexist within a narrow energy range in the $A \approx 70$ region. This can be understood qualitatively from the single-particle level scheme, in which large shell gaps occur for proton and neutron numbers 34,36 , and 38 , both for prolate and oblate deformations. More detailed mean-field calculations, e.g. Hartree-Fock calculations using the Skyrme force [1], find two distinct minima at large oblate and prolate deformations, which are almost degenerate in the case of ${ }^{74} \mathrm{Kr}$. Consequently, one may expect to find low-lying $0^{+}$states in this region that are likely to be metastable, so called shape isomers.

Such an isomeric low-lying $0^{+}$state was recently found in ${ }^{72} \mathrm{Kr}$ by delayed conversion-electron spectroscopy after fragmentation of an energetic $(70 \cdot A \mathrm{MeV}){ }^{78} \mathrm{Kr}$ beam [2]. This shape isomer is located below the first $2^{+}$ state and consequently decays exclusively via a non-radiative $E 0$ transition to the ground state. The measured lifetime of 38(3) ns [2] results in a very enhanced electric monopole strength of $\rho^{2}(E 0)=(72 \pm 6) \times 10^{-3}$, indicating a significant shape change and a strong mixing of the wave functions. The observation of the shape isomer in ${ }^{72} \mathrm{Kr}$ extended the systematics of lowlying $0^{+}$states in the light $\mathrm{Kr}$ isotopes to the $N=Z$ line, as is illustrated in Fig. 1. The excitation energy of the $0_{2}^{+}$states decreases towards the more neutron-deficient isotopes, finds a minimum in ${ }^{74} \mathrm{Kr}$, and increases again in ${ }^{72} \mathrm{Kr}$. This has been interpreted as a lowering of the oblate configuration, which crosses the prolate configuration in ${ }^{74} \mathrm{Kr}$ and becomes the new ground-state configuration in ${ }^{72} \mathrm{Kr}$. The $0_{2}^{+}$state in ${ }^{72} \mathrm{Kr}$ is then understood as the band head of the prolate rotational band. This scenario is supported by the observed $E 0$ strengths and by the mixing amplitudes of the prolate 
and oblate configurations in the ground states that were derived from an extrapolation of the regular rotational bands at higher spin [2]. This showed that the unperturbed $0^{+}$states in ${ }^{74} \mathrm{Kr}$ are practically degenerate and that the observed energy difference of the states is entirely due to the repulsion of the intrinsic states. However, although the scenario is conclusive, it is based only on indirect observations. In order to prove it, one has to measure static quadrupole moments, which give access to the sign of the deformation. Such measurements for unstable nuclei have become experimentally possible by performing Coulomb excitation of low-energy beams of radioactive isotopes.

\section{Low-energy Coulomb excitation of ${ }^{76} \mathrm{Kr}$ and ${ }^{74} \mathrm{Kr}$}

Coulomb excitation at energies well below the barrier populates collective states that are linked to the ground state predominantly by $E 2$ or $E 3$ matrix elements in a pure electromagnetic process. The interaction time between the projectile and target is long enough to populate higher-lying states in multiple steps. The electromagnetic interaction can be calculated with high precision including higher-order effects, which cause a difference in the Coulomb excitation cross section for different intrinsic shapes of the nucleus [3]. If the precision of the measurement is high enough, the diagonal matrix elements (and therefore the intrinsic shapes) can be extracted from the $\gamma$-ray yields originating from the various states measured as a function of the scattering angle.

Projectile Coulomb excitation of radioactive ${ }^{76} \mathrm{Kr}$ and ${ }^{74} \mathrm{Kr}$ beams was performed in experiments at the SPIRAL facility of GANIL. The secondary beams were produced by fragmentation of a $68.5 \cdot A \mathrm{MeV}{ }^{78} \mathrm{Kr}$ primary beam of $10^{12} \mathrm{pps}$ on a carbon target. The radioactive ions were extracted using the ISOL method and then re-accelerated to $4.5 \cdot A \mathrm{MeV}$. The intensity of the pure secondary beams was $5 \cdot 10^{5}$ pps for ${ }^{76} \mathrm{Kr}$ and $10^{4} \mathrm{pps}$ for ${ }^{74} \mathrm{Kr}$. A ${ }^{208} \mathrm{~Pb}$ foil of $1 \mathrm{mg} / \mathrm{cm}^{2}$ thickness was used as the secondary target. The scattered projectiles and the recoiling target nuclei were detected in a highly segmented annular silicon detector. This allowed the kinematic reconstruction of the events and the measurement of the Coulomb excitation cross section as a function of scattering angle. A continuous range of scattering angles between $24^{\circ}$ and $145^{\circ}$ in the center-of-mass frame was covered by either detecting the projectile or the target nucleus. Gamma rays were detected in the EXOGAM array, which comprised $7\left({ }^{76} \mathrm{Kr}\right)$ and $11\left({ }^{74} \mathrm{Kr}\right)$ clover detectors at the times of the experiments, respectively. The segmentation of both the silicon and the germanium detectors limited the Doppler broadening to $8 \mathrm{keV}$ for a $500 \mathrm{keV}$ transition. States up to the $8^{+}$of the ground-state band and several non-yrast states were populated in both the ${ }^{76} \mathrm{Kr}$ and ${ }^{74} \mathrm{Kr}$ experiments, as shown in Fig. 2 . The total $\gamma$-ray spectra ob- 

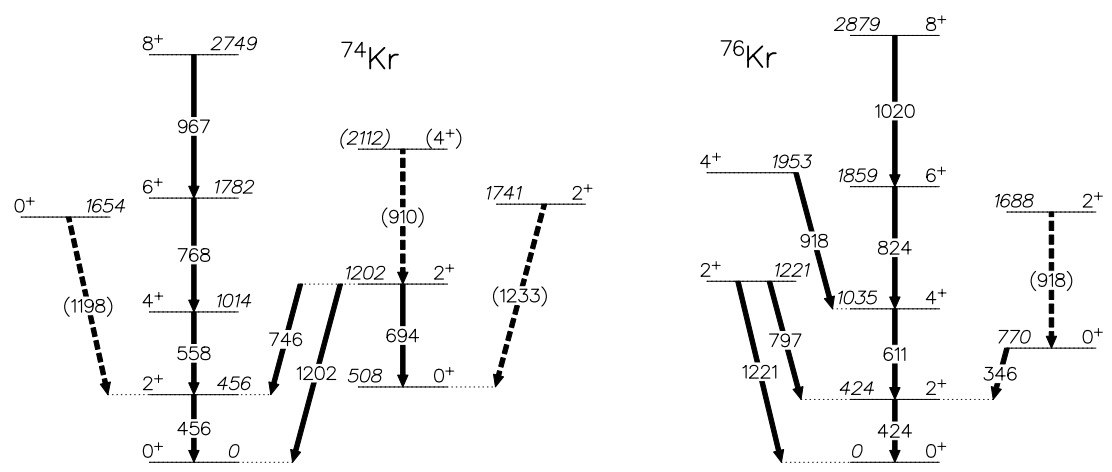

Fig. 2. Partial level schemes of ${ }^{74} \mathrm{Kr}$ and ${ }^{76} \mathrm{Kr}$ showing the observed transitions after Coulomb excitation. Transitions that could not be identified unambiguously are shown as dashed arrows.
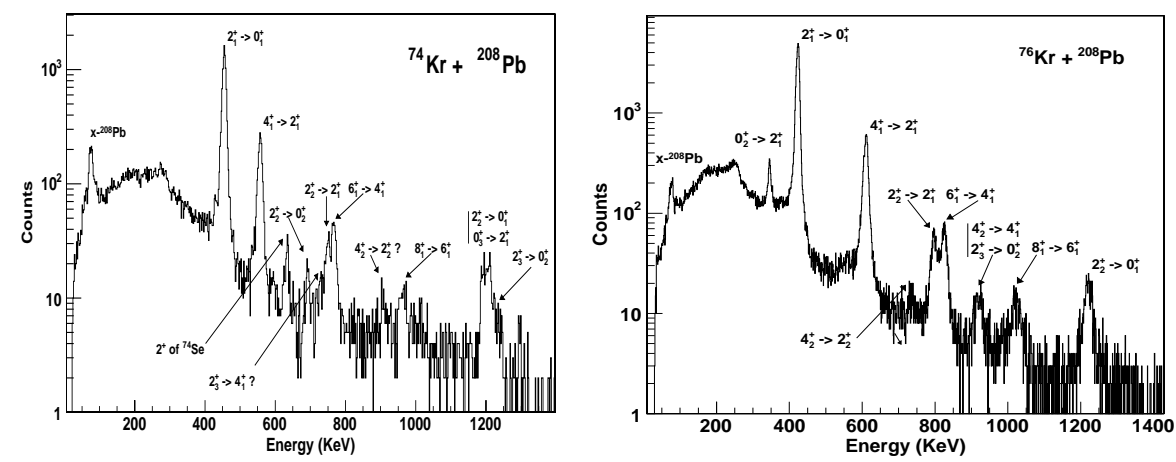

Fig. 3. Total $\gamma$-ray spectra observed after Coulomb excitation of ${ }^{74} \mathrm{Kr}$ (left) and ${ }^{76} \mathrm{Kr}$ (right). The spectra represent the integrated yields over the entire range of scattering angles covered by the silicon detector.

served in coincidence with scattered projectiles in the two experiments are shown in Fig. 3. The coincidence requirement eliminated the dominating background from the radioactive decay of the beam particles completely.

The total data sets of each experiment were divided into four subsets corresponding to different ranges of scattering angles. The four resulting $\gamma$-ray spectra for ${ }^{74} \mathrm{Kr}$ are shown in Fig. 4 . They illustrate how the excitation probability changes with the scattering angle. The $\gamma$-ray yields from the different subsets of data were analyzed with the code GOSIA [4]. Both transitional and diagonal matrix elements are found in a $\chi^{2}$ minimization by comparing the observed yields with the ones calculated from the matrix elements. Any known spectroscopic information such as lifetimes and branching ratios can be used in the multi-dimensional fitting procedure in addition to the $\gamma$-ray yields. 


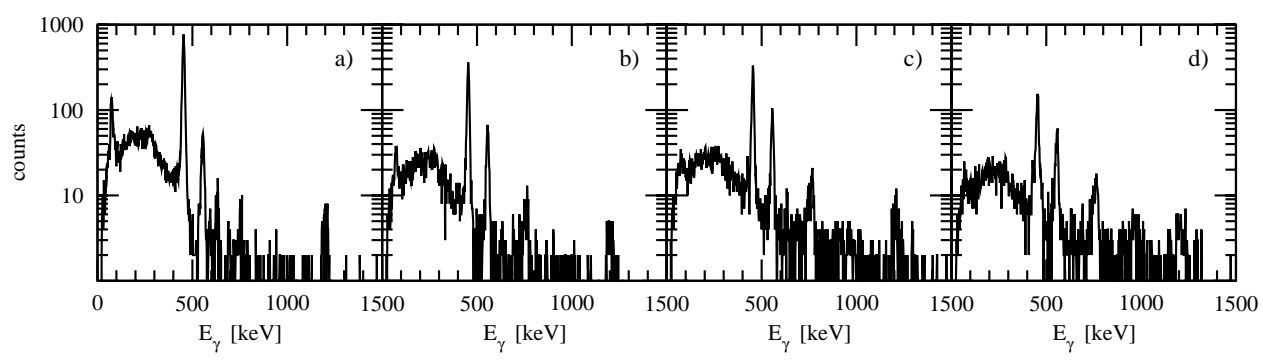

Fig. 4. Gamma-ray spectra observed after Coulomb excitation of the ${ }^{74} \mathrm{Kr}$ projectiles on ${ }^{208} \mathrm{~Pb}$ targets for different scattering angles (given in the center-of-mass frame): (a) $24^{\circ} \leq \theta_{C M} \leq 54^{\circ}$, (b) $54^{\circ} \leq \theta_{C M} \leq 74^{\circ}$, (c) $67^{\circ} \leq \theta_{C M} \leq 97^{\circ}$, (d) $97^{\circ} \leq \theta_{C M} \leq 145^{\circ}$

Constant values of $2.8 \mathrm{eb}$ were found for the transitional quadrupole moments in the ground-state band of ${ }^{76} \mathrm{Kr}$, with a precision of about $3 \%$ for the individual values. These results are in agreement with those obtained in a lifetime measurement [5]. The static quadrupole moments have positive sign, showing that the deformation of the ground-state band is prolate. The absolute values are consistent with the $Q_{0}$ moments inferred from the transitional matrix elements and have an uncertainty of about $10 \%$, leaving no doubt about the positive sign. This result represents the first measurement of static quadrupole moments in a radioactive-beam experiment. While transitional matrix elements were also found for non-yrast transitions, it was not possible to extract the diagonal matrix elements for any non-yrast states. Information from transitions above the state in question is essential for the measurement of static quadrupole moments. Such information could not be obtained sufficiently for the non-yrast states in ${ }^{76} \mathrm{Kr}$.

The data on ${ }^{74} \mathrm{Kr}$ are still under evaluation and the results presented here are preliminary. As for ${ }^{76} \mathrm{Kr}$, both transitional and diagonal E2 matrix elements were found for the ground-state band. The transitional matrix elements could be measured with a similar precision as in the ${ }^{76} \mathrm{Kr}$ case. However, the measured lifetime of the $4_{1}^{+}$state [6] cannot be reproduced when it is treated as a free parameter in the GOSIA fit. Using this lifetime as a constraint in the fit results in a distortion of the band and a significantly lower quadrupole moment for this transition. A new lifetime measurement will resolve this question. The static quadrupole moment for the $2_{1}^{+}$state is large and positive, confirming the prolate character of the ground-state band also for ${ }^{74} \mathrm{Kr}$. Even though the absolute value of the quadrupole moment might change somewhat depending on the outcome of the new lifetime measurement, a negative sign can be excluded. Since the $2_{2}^{+}$state in ${ }^{74} \mathrm{Kr}$ is lower in energy compared to ${ }^{76} \mathrm{Kr}$, results were also found for this 
state. We find a large $E 2$ strength for the $694 \mathrm{keV}$ transition to the $0_{2}^{+}$state and a negative intrinsic quadrupole moment, confirming the oblate character of this rotational band that had been suggested for this structure [7]. The absolute value of the static quadrupole moment of the $2_{2}^{+}$state depends on the yield observed for the $4_{2}^{+} \rightarrow 2_{2}^{+}$transition. The $4_{2}^{+}$state, however, is not known. The most consistent fit of the data is achieved if the $910 \mathrm{keV}$ transition, that cannot be attributed otherwise, is assumed to originate from the $4_{2}^{+}$state. The observed $\gamma$-ray yields cannot be reproduced if the opposite (prolate) sign is assumed for the $2_{2}^{+}$state. These results represent the first unambiguous experimental evidence for coexisting states of prolate and oblate deformation in this mass region.

\section{Intermediate-energy Coulomb excitation near ${ }^{72} \mathrm{Kr}$}

The intensity expected for a ${ }^{72} \mathrm{Kr}$ beam from SPIRAL is currently too low to perform a Coulomb excitation measurement at low energy. Instead, isotopes near the $N=Z$ line have been investigated at intermediate energies of $\sim 40 \cdot A \mathrm{MeV}$, where a low beam intensity can be compensated by the use of a thick secondary target. This method, however, populates only states that can be reached in a single-step excitation, and the precision is not sufficient to extract diagonal matrix elements. Secondary beams of radioactive ions were produced by fragmentation of a high-intensity ${ }^{78} \mathrm{Kr}$ beam of $73 \dot{A} \mathrm{MeV}$ on a $\mathrm{Ni}$ target of the SISSI device. The different species were separated in the LISE3 spectrometer and identified event by event by their time of flight through the spectrometer and their energy loss in a stack of silicon detectors. Two fast position-sensitive micro-channel plate detectors were used to track the beam. The ions were Coulomb excited on a $220 \mathrm{mg} / \mathrm{cm}^{2}$ thick ${ }^{208} \mathrm{~Pb}$ target which was surrounded by four EXOGAM clover detectors in a close geometry. A stack of two highly segmented annular silicon detectors, with thicknesses of 140 and $300 \mu \mathrm{m}$ was placed $42 \mathrm{~cm}$ behind the target. They were used to identify the scattered ions and to measure scattering angles between $\sim 1^{\circ}$ and $5^{\circ}$. The ions that passed through the target without interaction were identified and counted in a $300 \mu \mathrm{m}$ thick plastic scintillator and subsequently implanted into a second plastic scintillator that was tilted $45^{\circ}$ with respect to the beam axis. Two alcohol-cooled silicon detectors of $1 \mathrm{~mm}$ thickness and $50 \times 50 \mathrm{~mm}^{2}$ surface were used to look for isomeric conversion-electron decays from the implanted nuclei.

The main goal of the experiment was to identify the $2_{2}^{+}$state in ${ }^{72} \mathrm{Kr}$ and to measure the $B(E 2)$ values to the first and second $2^{+}$states. Even though the data analysis has only just started, it can already be concluded that the yield of $\sim 20$ pps for ${ }^{72} \mathrm{Kr}$ was not sufficient to achieve these goals. However, many other isotopes were produced simultaneously with higher 
yields, and new results can be expected for other isotopes in this region, e.g. for ${ }^{68} \mathrm{Se}$. Data have been analyzed so far only for ${ }^{72} \mathrm{Ge}$. The $\gamma$-ray spectrum in coincidence with scattered ${ }^{72}$ Ge ions is shown in the left part of Fig. 5, showing the $2^{+} \rightarrow 0^{+}$transition following Coulomb excitation. The first excited state in ${ }^{72} \mathrm{Ge}$ is known to be a $0^{+}$state at $690 \mathrm{keV}$ [8]. Since the ions are fully stripped of their atomic electrons when passing through the spectrometer and they regain electrons only when they are implanted, nuclei produced in the excited $0_{2}^{+}$state are trapped and can only decay after implantation. The $E 0$ decay between the two $0^{+}$states is therefore observed in the conversion-electron spectrum, shown in the right part of Fig. 5. The data for neighboring isotopes is under analysis.
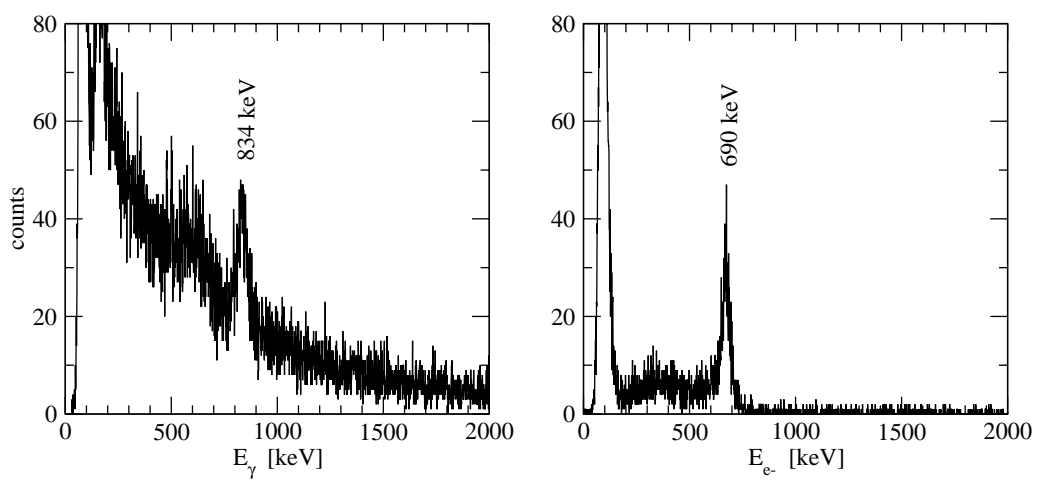

Fig. 5. left: Prompt $\gamma$-ray spectrum from the Clover detectors surrounding the target in coincidence with scattered ions identified as ${ }^{72} \mathrm{Ge}$, showing the $2^{+} \rightarrow 0^{+}$ transition after Coulomb excitation. right: Conversion-electron spectrum from the Silicon detectors showing the $E 0$ decay from the excited $0^{+}$state after implantation.

\section{REFERENCES}

[1] P. Bonche et al., Nucl. Phys. A443, 39 (1985); and P.H. Heenen, priv. communication

[2] E. Bouchez et al., Phys. Rev. Lett. 90, 082502 (2003)

[3] K. Alder, A. Winther, "Electromagnetic Excitation, Theory of Coulomb Excitation with Heavy Ions", North-Holland, 1975

[4] T. Czosnyka, D. Cline, C.Y. Wu, Bull. Amer. Phys. Soc. 28, 745 (1983); GOSIA users manual, University of Rochester, UR-NSRL-305 (1991)

[5] B. Wormann et al., Nucl. Phys. A431, 170 (1984)

[6] J. Roth et al., J. Phys. G: Nucl. Phys. 10, L25 (1984)

[7] F. Becker et al., Eur. Phys. J. A 4, 103 (1999)

[8] W.-T. Chou and M.M. King, Nucl. Data Sheets 73, 215 (1994) 